\title{
Integration Framework for Information Systems Coexistence within Organization
}

\author{
Tefo Sekgweleo ${ }^{1, *}$, Johnson Mbuka Billawer ${ }^{2} \&$ Lydia Hamunyela $^{3}$ \\ ${ }^{1,2,3}$ Polytechnic of Namibia, Windhoek, Namibia \\ *Correspondence: Polytechnic of Namibia, Windhoek, Namibia. E-mail: ts330ci@gmail.com
}

Received: September 8, 2015 Accepted: October 22, 2015 Online Published: December 2, 2015

doi:10.5430/mos.v3n1p17 URL: http://dx.doi.org/10.5430/mos.v3n1p17

\begin{abstract}
The demand for better services by customers force, organisation to keep evolving. This requires organisation to acquire several information systems (IS) hence the integration of these IS become a challenge. To achieve a successful IS integration, it is important to understand critical factors that impact integration. Therefore, the study investigated the impact of having several information systems in silos, as well as understanding the factors that influence the IS integration and developed a reference framework to aid organisation in the integration of IS. The interpretative approach was employed in the empirical data.
\end{abstract}

Keywords: Information systems (IS); Integration

\section{Introduction}

Organisations rely on the use of information systems to be efficient and to remain competitive. On the other hand employees rely on information systems to complete their daily activities. Many organisations have embraced information systems to increase productivity, profits and save costs (Watson, Boudreau, \& Chen, 2010). The more the organisation grows the more they develop or purchase more information systems. As a result, some of the information systems are have to communicate with each in order to address the holistic needs of the organisation. The most important thing facing the organisation is to accomplish its strategy. According to (Pappa \& Stergioulas, 2008) information system is developed to support certain functions of the business which are aligned with the business strategy.

When having multiple information systems with the organisation, centralization of information may be required across various business units/departments. However, if these systems are not integrated it may be difficult for the employees to access information from other departments. Hobbs, Adelstein, O'hara, \& Null (2008) posit that inadequate human system integration increases the overall complexity of the system, increases the time needed to perform tasks, complicates training and maintenance, while decreasing the capabilities of the system. Monteiro (2003) argues that the aim of integration is to eliminate redundant operations, sorting out ambiguity and cut back secondary administrative overhead. The aim of system integration is to ensure that the systems are able to communicate with each, redundancy does not occur and employees are able to access information across the organisation. According to Shore (2006) the purpose for system integration is to create a shared information environment that supports the delivery of products or services.

The research question was how can organisation integrate multiple information systems within its environment? This includes examining the impact of having several information systems in silos, as well as understanding the factors that influence the systems integration.

\section{Literature Review}

\subsection{Information Technology}

Information Technology (IT) is defined to be a set of entities and activities that relates to computer systems, such as hardware and applications. The process of development, implementations, maintenance and, supports are all part of 
Information Technology (Shroff, 2010). Information, Communication and Technology has changed how people view and understand the world, and how they relate to each other (Jordan, 2012). In recent years, the world has experienced a much growth in businesses and communication because of Technology.

Many individuals and organizations make use of IT primarily to enhance their processes and activities. Carr (2003) stated that Information Technology has become the backbone of economy and support organizations to grow and successfully attain goals and objectives. Along the same line of debate, Thong \& Yap (1995) argued that information technology provides an opportunity for businesses to improve their efficiency and effectiveness, and even to gain competitive advantages.

According to Boar (2002), the purpose of IT is to provide a robust resource for the building, compounding, and sustaining of competitive advantage for the enterprise. Cost reduction, expedited decision making, and improved productivity, while of course important, are but specific instances of this greater purpose. In the new millennium, the purpose of IT is to be the foundation of competitive advantage for the business.

The rapid diffusion of information technology (IT) is a direct consequence of the swift decline in the price of computer-related equipment, which has led to a vast and continuing substitution of IT equipment for other forms of capital and labor (Venkatesh et al., 2003). Matthews (2007) stated that Information Technology is acknowledged to play an important role in the growth of enterprises by contributing directly to profitability and by providing foundations for the evolution of operations from a micro to a medium level.

\subsection{Information Systems}

The study of information systems is normally premised on the assumption that information systems are socio-technical systems that encompass both technical and social variables. An information system is a constellation of technologies, including computers (hardware), communication protocols, and network technologies that support various ways of handling, communicating, consolidating and processing information, and in this way allow organizations (and even societies) to change the ways in which they operate (Cordella \& Iannacci, 2011).

According to Chen, Mocker, Preston, \& Teubner, (2010), information systems within organizations comprise of the information technology infrastructure, data, application systems and personnel that adopts IT to deliver information and communications services. As a result, organizations desperately need well-trained information systems specialists and technology-savvy managers. The information system should be able to be integrated within the organization's culture as well as supporting their working practices (Maguire \& Redman, 2007). Employees simply cannot effectively function without the use of information systems within their organizations.

\subsection{System Integration}

Organisations usually purchase/develop information systems (IS) to replace manual ways of performing duties or improving current ways of doing things. At times it happens that organisations acquire multiple information systems to be used by various departments. This leads to various challenges such as certain systems duplicating tasks, causing data redundancy and information not stored in multiple places. In order to harmonize the operation and interaction amongst these IS the integration issue becomes important. The term enterprise integration or system integration reflects the capability to integrate a variety of different system functionalities (Lee, Siau, \& Hong, 2003).

Integrating different systems in an organization can be very challenging. Doan \& McCann (2003) posit that data integrated systems provide a uniform query interface to a multitude of data sources, thereby freeing the user from the tedious task of interacting and combining data from the individual sources. A study conducted by Tatari \& Skibniewski (2011) identified the following critical factors for systems integration: top management support and commitment; availability of financial resources, clear systems integration strategy, goals, and vision; adequate ERP vendors support, Information Systems department competence, user training, and clear allocation of responsibilities. Sandoe, Corbitt, \& Boykin (2001) classified obstacles to integration into three categories as people-related obstacles, process-related obstacles, and technology-related obstacles.

There are certain features that need to occur when integrating information systems. They include integration testing and performance testing. Integration testing is a process of testing related programs, modules or units of code to confirm that multiple parts of the system interact according to the system design (Tewari \& Misra, 2010). Software system integration refers to the practice of combining individually tested software components into an integrated whole. Usually when information systems are integrated, the new hardware is purchased to accommodate all the systems to be integrated. Therefore, performance testing is required to ensure that all the integrated systems perform as expected on the new hardware. The aim of performance testing is to expose problems that are revealed when the system is run under specific workloads (Gan, 2006). The performance tests are used to evaluate and understand the 
volume of data if users are increased on the system. This is particularly important for identifying bottlenecks in high usage applications.

\subsection{Systems Implementation}

Prior to systems implementation, intense software testing has to occur to ensure that defects are eradicated. The intended system has to fulfill the user requirements and be accepted by the users. User acceptance testing is conducted with the user to ensure that the system functions as specified in the requirement specifications (Tan \& Tan, 2010). Once the users have accepted the new system, it is then implemented into production. However, there are various implementation strategies that can be followed. They include big bang and incremental (Gebauer \& Lee, 2008). Big bang refers to replacing the old system with the new one at once. Incremental refers to implementing the new system in stages.

\section{Research Methodology}

The purpose of this study is to develop integration framework for systems coexistence within the organisation. In order to achieve this goal, various methods and approaches were employed. They include the qualitative method, case study research method and data collection approaches. A case study aims at investigating cases which may be an organisation, department, project, information system or even a person. According to Baxter \& Jack (2008), case study enables a researcher to explore or describe a phenomenon in context using a variety of data sources.

The data was collected through semi-structured interviews. The semi-structured interview involves prepared questioning guided by identified themes in a consistent and systematic manner with probes designed to elicit more elaborate responses ( $\mathrm{Qu} \&$ Dumay, 2011). This technique enables the interviewers to gather in-depth information by probing the interviewees on the subject being investigated. An interpretative approach was used for data analysis. According to Kura \& Sulaiman (2012) this approach seeks to understand knowledge based on social reality through detailed understanding and interpretation of meaning of events and specific life experiences.

\section{Data Analysis}

\section{What is the impact of having several information systems in silos within the organisations?}

Organisations evolves and keep growing as customers are demanding for better services, it has brought a situations where the organization have information systems that are in silos. These systems are in silos because they are not integrated with other platforms and they are still used by some users, and they are still meeting certain requirements within the organization. Although there are critical and main systems within the organizations, there are now those standalone information systems which are referred to as silos, According to one of the interviewees:

"There are many information systems that have been implemented in the past, which include critical systems such as BSS, our billing platform and also OSS, however there very many IS that are not integrated with the critical systems such as Ozeki, IP Plan, Wiki, and very many systems" (TC_001, P2: 34 - 35).

The systems that are implemented in silos as examples listed above are still useful and required by the organizations for various functions. However, for the systems to keep in silos could have some impact to an organization directly or indirectly.

\section{Why are systems implemented in silos?}

Some systems have been in the organization for some times and they are still required by users to meet specific functions, then the business would allow such silos systems to co-exist with other platform although they are not integrated. There are times that a small section such as Public Relations or Audit would want specific information systems which only they will use and in this case it will be silo systems as it affect no other systems within the organization. According to one of the interviewees:

"For new applications that business wants, any section simply logs a change request. What happens then is that change request is assigned to a certain domain for analysis, so we will specifically look at all the changes, so when the analysis is done, the approval is made based on the requirement, and a project is assigned to bring in the new system" (TC_003, P11: 343 -346).

There are many reason that causes several systems to be implemented in silos, although having systems in silos is regarded as a negative aspect on Information Technology today, the Information systems on that silos platform is still required and meeting a specific need but the silos came as results of many factors. One of the factors highlighted 
is that when implementing solution without taking time to plan and consider all aspects of implementation and testing have resulted in silos.

"Sometimes we do not take time to implement systems, and everything is done in a rush, that at the end of the day some systems end up not communicating to any other systems" (TC_001, P6: 180-181).

Prior to that, when there is no time to consider the technology and how it fits in with all other systems contributed to high level of silos. Another factor apart from time was based on understanding how technology and systems fits in with others, because there is no clear standards and specified guidelines to be followed when bringing in a new system and how to regards old systems. One of the interviewee stated that:

"The other one is the lack of understanding of business or sometimes you find that's a lack of understanding of business and your business requirements are not clearly specified because of misunderstandings about what is important and what is not important and if that is discovered late in the systems development life cycle then you stand a chance of having to redo the whole solution" (TC_001, P4: $120-124)$.

Prior to making any changes to the system, it is vital for the individual to understand the organisational strategy, the new technology to be adopted and how the new/upgraded system needs to work. If the above mentioned factors are not taken into consideration then productivity within the organisation may be negatively impacted due to lack of understanding. As a result, the organisation may incur a huge loss. Lack of understanding of technology when introducing new systems have created a number of silos, since there was no understanding how these systems could be integrated with the existing systems, adding to that another interviewee expressed his concerns by stating that:

"From the technology perspective if one doesn't look at how the technology fits into your strategy you will have problems where there is many different systems in the organisation that cannot talk to each other and as such you end up integrating the different systems incorrectly because you did not look at how these systems will be able to talk to each other from the beginning and sometimes it even leads to some systems being thrown out because they were just done incorrect in the first place, it's impossible to integrate them to the rest of the organisation"(TC_001, p4:101-107).

There are times that systems are implemented by the third party or vendors who do not have overall understanding of the existing systems within the organizations, so those new systems may be speaking a different technology language than the existing systems cannot understand, and that lead to silos. According to one of the interviewee:

"I think in some cases vendors don't understand the issues that affect the systems especially the technology in the existing one, so during the testing phase things are ok, in the production environment things are not working" (TC_002, P9: 270-272).

\section{What is the implication to the organization (and to the specialists, such as developers and support team)?}

Although this silos systems are needed and still required for various functions, however from the infrastructure's perspective there several implications to the business when having information systems all over the place, implications are such as maintenance, instead of systems administrator to focus on critical systems, they may take more time attending to several systems here and there, instead of focusing on overall administration and on critical information systems to the business. According to one of systems administrator interviewed:

"The system administration role is to make sure that the systems are easy to maintain and fits within the current infrastructure or operational way of doing things, however with many silos the maintenance becomes complex and requires extra human resource" (TC_001, P2: 46 -48).

Prior to such implications of systems administration and maintenance, there is a cost implication on the business on cost aspects, more systems support will be required and that means more labor costs, and not only labor cost, having many systems on silos means more resources to spend on such systems as they taking up space, operational costs, hardware and software costs, cabling and power and network costs.

"No after the investigation was complete and when going back to the planning phase we realized that even if this is important requirement, it can cost the organization more than was anticipated" (TC_0010, P54: $1687-1688)$.

\section{How can organisation integrate multiple information systems?}

Some employees who were participating in the integration of systems highlighted that formalized processes and standards were followed in the organization. 
"We are working according to SDLC and our organisation has got its own customised process and we call it the solution value change so every project inception either start with a business case or a change request that is logged at our change forum so it has the integrated change control forum so all new enhancements or new applications that business wants they need to log a change request with the ICCF” (TC_003, P11:341-346).

"We adopted ETOM - a business process framework that make sure that the fulfillment assurance and billing is segregated well enough to allow the company to have different points of integration either at BSS layer which is your billing system or at the integration layer or at the OSS layer which is your fulfillment" (TC_001, P2:32-35).

\section{What is the importance of the integrating multiple systems?}

Some organisations make use of a number of systems to perform various types of activities. The aim of embracing these systems is for the organization to be effective and efficient in the products or services it renders.

"It is important for these systems to be able to speak to each other" (TC_001, p6:181-181).

\section{What are the implications?}

There are various implications which could impact business negatively if integration is not performed properly within the organisation.

"Most of the time it's time, I think because of not enough time incorrect solutions get implemented because things are done too quickly to make a particular problem disappear that is one of the factors. The other factor I would say is politics, in any organisation different people see different ways of going about the same thing so managing peoples' expectations both from the business requirement as well as technology requirement I would say it can be one of the factors that affect or could pose a possible risk to most systems project. The other one is the lack of understanding of business or sometimes you find that's a lack of understanding of business and your business requirements are not clearly specified because of misunderstandings about what is important and what is not important and if that is discovered late in the systems development life cycle then you stand a chance of having to redo the whole solution" (TC_001, p4:114-124).

\section{What are the factors that influence the systems integration?}

The technological world keeps on evolving. As a result, the systems currently used within organisations are directly or indirectly affected by such evolvement. Therefore, some of these systems become absolute and require replacing or upgrading.

"When introducing a new technology to the environment that already has certain systems in place, those systems have to be configured in order to be compatible to the new technology that is being adopted for business to continue as usual or else it will be difficult for business users to carry out their daily duties" (TC_001,p2:49-54).

As a result of forever changing technology individuals within the organisation are able to learn new things by themselves. It is vital for individuals to keep up with latest technology by exploring it and up skilling themselves through training.

"The enlighten of the way technology is progressing towards infrastructure is just a platform for applications to run on and I think the concept of focus on infrastructure is also moving towards a much more modular view if you look at the way virtualisation has come in and the way infrastructure as a platform as a service is starting to take the market and you then have a scenario where infrastructure is not so much of a concern to you because you can plug in and plug out anything in your infrastructure layer be it you want a database is no longer about finding a server somewhere that is well documented to be a database but in five minutes you can deploy a database and whatever your application requires will be provided in five minutes as compared to go and buy software and hardware, etc" (TC_001, p5:144-152).

Exploration of new technology also enables individuals to discover new and relevant ways of doing things which adds value to their organisations.

"Right frameworks allow agility in the business especially on the systems perspective to be able to develop systems in a much more modular way of whilst making sure that all the modules or components of the 
system are able to speak to each other" (TC_001, p6:178-181).

As the technology keeps on changing, competition become intense amongst organisations. As a result, organisations are forced to keep up in order to survive.

"It is very important to stay abreast new technologies and also if there is better technologies out there that we can use to improve our applications to user friendliness, the performance we need to keep abreast of that because we need to implement it we can't have out dated software and hardware you know the thing is not performing, it is slow no we need to stay on top of what is going on out there because I mean we are competing in the competitive world we need to compete with our rivals their things are faster and fancier so we need to get there as well or else nobody will use our applications" (TC_003, p15:478-485).

\section{Findings}

The findings from the analysis of the data which was obtained from the organisation used in the study is presented in this section. After carefully analysing the data, the following factors were found to be critical to the integration of systems in the organisation: communication, change, lack of understanding, exploration of new technology and improvement.

\subsection{Communication}

Communication is very crucial within an organisation. When the organisation is embarking on information systems projects, there must be effective communication amongst the project team members involved in developing, implementing and integrating information systems. Similarly, all concerned employees in the organisation need to be kept informed regarding a new change or innovation to be introduced. All stakeholders must understand the communication channels and the media being employed to disseminate information. Lack of communication can lead to confusion and misunderstandings in an organisation.

\subsection{Change}

Due to the fact that technology keeps on evolving, it is vital for organisations to keep up with it. Therefore, organisations are forced to improve on how they do things in order to compete with its competitors. As a result, the adopted information systems are affected and they require change. Change comes with its own challenges because these information systems will have to be upgraded or new ones brought in. As a result, integration of those information systems is also affected. Then employees involved with the mentioned changes will be required to implement those changes.

\subsection{Lack of Understanding}

The integration of information systems can be achieved through a combination of technical and organisational process innovations. On a technical level, integration requires the use of industry-standard common databases and communication protocols. Similarly, on the organizational process level businesses need to simplify and streamline its organizational processes. The lack of understanding on how a business operate subsequently attribute to wrong business requirements being defined. This, would in turn results in wrong information systems implemented in the organisation and that can even be difficult to integrate such systems.

\subsection{Exploration of New Technology}

The best tool for handling lack of understanding will be to explore the new technologies and learn how they work. Training will as well be required for up skilling the employees involved with the change. External help may also be required to solve other problems. Once the technology is understood challenges encountered are then resolved. As a result, the organisation will be in a position to continue operating and competing with its rivals.

\subsection{Improvement}

In today's modern world organisations operate in a very competitive environment. Therefore, in order for an organisation to achieve and retain its competitive advantage it must keep on improving how it is doing things. Improvement in this context refers to new technology that will enable the organisation to offer quality products and render the best service.

\section{Interpretation of the Findings}

The interpretation from the analysis of data and the findings revealed some factors which influence the integration of 
information systems in the organisation. Those factors include communication, transformation, poor implementation, training, and innovation.

\subsection{Communication}

Although different communication channels were employed, the analysis of data revealed that effective communication was lacking amongst the IT project team members as well with external parties, resulting in misunderstanding of information. This is evident as some employees involved in the systems development and integration projects seem to not understand business requirements. This was due to lack of communication between the business analysts, software developers and software vendors. This resulted in the implementation of some information systems not suited for the organisation and are difficult to maintain and integrate with other systems.

\subsection{Transformation}

Transformation in the organisation can be a complex process that needs to be managed properly. Based on the analysis of data and the findings revealed that there were issues encountered with introducing new information systems in the organisation. Firstly, due to change the organisation acquired several information systems that became difficult to manage. Secondly, not all stakeholders seem to be on the same page with regard to changes introduced in the organisation. The communication factor pointed out earlier is critical here. Any change in the organisation need to be communicated and all stakeholders must be involved in the process in order to gain buy-in from all stakeholders.

\subsection{Poor Implementation}

Whenever the organisation is deploying the information system, a proper planning that include the whole process of systems implementation need to be employed, as highlighted on the analysis of data collected, the finding reveals that poor implementation of information systems has contributed to information systems implemented in silos within the organisation, it is also highlighted and found out that testing phase is mostly overlooked to ensure that the systems is meeting the required functions. Another factor that is identified contributing to poor implementation is of time, most of the time the business want to deploy a system so much in a hurry without following all procedure and planning for the entire implementation to be effective and accurate.

\subsection{Training}

As the technology advancing so rapidly, employees in an organisation must keep abreast with new developments. In order to eliminate lack of understanding and know how employees in an organisation must be exposed to these new technologies for them to explore and learn how they work. Therefore, training is critical to ensure that employees as well as those involved in the development and implementation of systems acquire the necessary skills and knowledge that will help them execute their roles successfully.

\subsection{Innovation}

In order for an organisation to maintain a competitive advantage above its competitors it has to be innovative. This implies that an organisation must continue introducing new ideas and practices to improve its operations. Thus, development and implementation of new information systems must be communicated to all stakeholders.

\section{Framework}

Based on the analysis and findings from the case study, a framework was developed. The framework is aimed at guiding organisations in the successful integration of separate information systems. Based on the analysis and discussions above influential factors for the integration of information systems can be classified in three main categories: people-related factors, process-related factors, and technology related factors. 


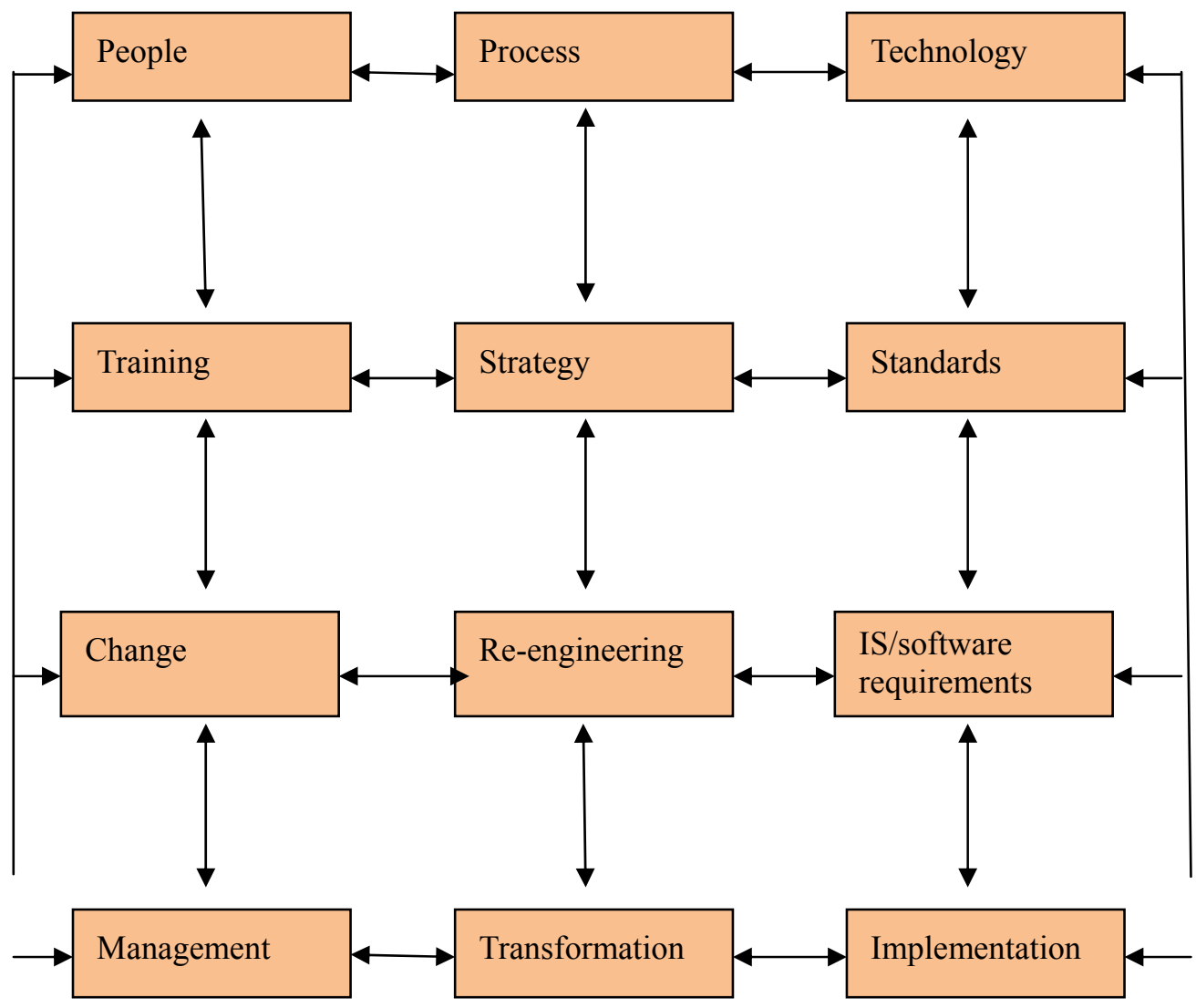

Figure 1. Integration Framework for Information Systems Integration

People/employees play an important role in the organization as they are involved in performing various roles and handling the information technology. Some of these roles could include the development, integration and implementation of information systems. Thus, management must ensure that adequate training is provided to enable employees' development. It is management responsibility to develop effective strategies that support training of the employees.

For the employees to participate in the different roles they need to understand the business processes. A clear understanding of business process enables employees to understand business operations and requirements. Management must clearly distinguish between the business and IT strategies so that the business units and the IT unit understand each other. Businesses are not static and do not operate in isolation and this call for business process improvement. The results of re-engineering may suggest a new technology or an upgrade of existing information systems.

Therefore, the transition from the current state to the new state needs to be managed carefully. Management must ensure that change in an organisation is managed properly to avoid employees' resistance. It is important that the development, integration and implementation of information system conform to standards set within the organization.

\section{Conclusion}

Organizations develop information systems to meet important business objectives such as improving competitiveness, increasing productivity and efficiency, accelerating growth, supporting innovation and reducing costs. Information system is the key to delivering the information and intelligence needed to improve innovation. Therefore, by investing in data storage systems and secure communication networks, the organization give its employees access to 
the information they need. As a result, a good information system can be used not only for the storage of electronic data alone but to help the organization compete both local and internationally with its counterparts in order to maximize profits. It is vital for organizations to integrate its information systems because that enables them to function effectively and efficiently. It helps the organization to synchronize all the information systems they are running. It also assists the organization to get rid of information systems duplication and data redundancy. As a result, the organization will save costs as it will not be necessary for it to develop any additional information systems.

\section{References}

Baxter, P., \& Jack, S. (2008). Qualitative case study methodology: Study design and implementation for novice researchers. Qualitative Report, 13(4), 544-559.

Boar, B. H. (2002). The art of strategic planning for information technology $\left(2^{\text {nd }}\right.$ ed.). John Wiley \& Sons.

Carr, N. G. (2003). IT Doesn't Matter. Harvard Business Review, 81(15), 41-49.

Chen, D. Q., Mocker, M., Preston, D. S., \& Teubner, A. (2010). Information Systems Strategy: Reconceptualization, Measurement, and Implications. MS Quarterly, 34(2), 233-259.

Cordella, A., \& Iannacci, F. (2011). Information systems and organizations. United Kingdom: University of London Press.

Doan, A., \& McCann, R. (2003). Building Data Integration Systems: A Mass Collaboration Approach. $18^{\text {th }}$ International Joint Conference on Artificial Intelligence (IJCAI).

Gan, X. (2006). Software Performance Testing. ACM Computing Classification System, 1-9.

Gebauer, J., \& Lee, F. (2008). Enterprise system flexibility and implementation strategies - Aligning theory with evidence from case study. Journal Information Systems Management archive, 25(1), 71-82. http://dx.doi.org/10.1080/10580530701777198

Hobbs, A., Adelstein, B., O'hara, J., \& Null, C. (2008). Three principles of human-system integration. Proceedings of the 8th Australian Aviation Psychology Symposium. Sydney, Australia.

Jordan, J. M. (2012). Information, Technology, and Innovation: Resources in a Connected World. John Wiley \& Sons. http://dx.doi.org/10.1002/9781119203056

Kura, B., \& Sulaiman, Y. (2012). Qualitative and Quantitative Approaches to the Study of Poverty: Taming the Tensions and Appreciating the Complementarities. Qualitative Report, 17(34), 1-19.

Lee, J., Siau, K., \& Dong, S. H. (2003). Enterprise integration with ERP and EAI. Association for Computing Machinery, 46(2), 54-60. http://dx.doi.org/10.1145/606272.606273

Maguire, S., \& Redman, T. (2007). The role of human resource management in information systems development. Management Decision, 45(2), 252-264. http://dx.doi.org/10.1108/00251740710727278

Matthews, P. (2007). ICT assimilation and SME expansion. Journal of International Development, 19(6), 817-827. http://dx.doi.org/10.1002/jid.1401

Monteiro, E. (2003). Integrating health information systems: A critical appraisal. Methods Inf Med, 42(4), 428-432.

Pappa , D., \& Stergioulas, L. K. (2008). The emerging role of corporate systems: An example from the era of business process-oriented learning. International Journal of Business Science and Applied Management, 3(2), $38-48$.

Qu, S. Q., \& Dumay, J. (2011). The qualitative research interview. Qualitative Research in Accounting \& Management, 8(3), 238-264. http://dx.doi.org/10.1108/11766091111162070

Sandoe, K., Corbitt, G., \& Boykin, R. (2001). Enterprise Integration. New York: John Wiley \& Sons.

Shore, B. (2006). Enterprise in across the globally disbursed service organization. Association for Computing Machinery, 49(6), 102-106. http://dx.doi.org/10.1145/1132469.1132475

Shroff, G. (2010). Enterprise Cloud Computing: Technology Architecture, Applications. New York: Cambridge University Press. http://dx.doi.org/10.1017/CBO9780511778476

Tan, W. K., \& Tan, C. H. (2010). Teaching Information Systems Development via Process Variants. Journal of Information Systems Education, 21(2), 159-172. 
Tatari, O., \& Skibniewski, M. J. (2011). Empirical Analysis of Construction Enterprise Information Systems: Assessing System Integration, Critical Factors, and Benefits. Journal of Computing in civil engineering, 25(5), 347-356. http://dx.doi.org/10.1061/(ASCE)CP.1943-5487.0000096

Tewari, A., \& Misra, A. K. (2010). Testing Manners and Technologies: An Analysis. International Journal of Computer Applications, 3(9), 17-22. http://dx.doi.org/10.5120/768-1075

Thong, J. Y., \& Yap, C. S. (1995). CEO Characteristics, Organizational Characteristics and Information Technology Adoption in Small Business. Omega, 23(4), 429-442. http://dx.doi.org/10.1016/0305-0483(95)00017-I

Venkatesh, V., Morris, M. G., Davis, G. B., \& Davis, F. D. (2003). User acceptance of information technology: Toward a unified view. Management Information Systems Quarterly, 27(3), 245-278.

Watson , R. T., Boudreau, M., \& Chen, A. J. (2010). Information Systems and Environmentally Sustainable Development: Energy Informatics and New Directions for the IS Community. Management Information Systems Quarterly, 34(1), 23-38. 